\title{
Monitoring metal ion leaching in oil-ZnO paint systems with a paramagnetic probe
}

\author{
Alessia Artesani ${ }^{\star^{1,2}}$, Laurent Binet ${ }^{3,4}$, Francesca Tana ${ }^{5}$, Daniela Comelli ${ }^{1}$, Luigi De Nardo ${ }^{5}$, Austin Nevin ${ }^{6,7}$, Nadia $^{3}$ \\ Touati $^{3,4}$, Gianluca Valentini ${ }^{1}$, Didier Gourier ${ }^{3,4}$ \\ 1. Politecnico di Milano, Physics Department, Milano, Italy \\ 2. Center for Cultural Heritage Technology (CCHT), Italian Institute of Technology (IIT), Italy \\ 3. Chimie-ParisTech, PSL University, CNRS, Institut de Recherche de Chimie-Paris (IRCP), Paris, France \\ 4. Centre de Recherche et de Restauration des Musées de France (C2RMF), Palais du Louvre, Paris, France \\ 5. Politecnico di Milano Chemistry, Materials and Chemical Engineering Department "G. Natta", Milano, Italy \\ 6. Istituto di Fotonica e Nanotecnologie - Consiglio Nazionale delle Ricerche (IFN-CNR), Milano, Italy \\ 7. Department of Conservation, University of Gothenburg, Gothenburg, Sweden
}

\begin{abstract}
Zinc oxide (ZnO), used as a pigment since the $19^{\text {th }} \mathrm{C}$., is highly reactive when mixed with drying oils. Indeed, the combination of metal-based pigments and drying oils may react to form metal complexes in paint, which may lead to the aggregation of metal carboxylates or soaps. Whereas the mechanism and chemistry behind metal soap formation has been studied in depth, little research has focused on the changes that affect the inorganic pigment particles. In this work, we concentrate on the first phase of these reactions and monitor the evolution of an oil-ZnO paint system through Electron Paramagnetic Resonance (EPR) spectroscopy. By employing $\mathrm{Cu}^{\prime \prime}$ as a paramagnetic probe, the progression of metal ion leaching from $\mathrm{ZnO}$ is followed through the switching of $\mathrm{Cu}^{\text {Il }}$ from a silent tetrahedral coordination in $\mathrm{ZnO}$ bulk to an EPR active pseudo-octahedral coordination. Complementarily, the effective concentration of dissolved or extracted $\mathrm{Zn}$ and $\mathrm{Cu}$ is quantified through Inductively Coupled Plasma-Optical Emission Spectrometry (ICP-OES). The experiment probes the spontaneous metal ion release in oil-based paint films and demonstrates that the kinetics of this phenomenon proceeds with a very steep increase in the first hours after mixing. This is followed by the saturation of the grow rate after a few days that we ascribe to the passivation of the $\mathrm{ZnO}$ pigment surface by carboxylate groups, which hinders the further leaching of metal ions, leading to a steady-state before the complete hardening or oxidation of the paint film.
\end{abstract}

\section{Manuscript}

\section{Introduction}

Curators and scientists are acutely aware of the importance of chemical reactions between pigments and binders since these govern the stability/instability of oil paints. Among modern pigments considered to be reactive in drying oil media, zinc white $(\mathrm{ZnO})$ is one of the most alarming. In $\mathrm{Zn}$-based paint, it has been shown that the reaction between pigment particles and drying oils leads to the formation of a metal carboxylate network (1)(2)(3). While during the initial stage this network results in the advantageous rigidity of paint film, in the longterm the crystallization of metal carboxylate complexes can be a problematic issue because of the formation of metal soaps that may compromise the adhesion and cohesion of paint (3)(4)(5). Despite the wide consensus on the principal chemical reactions leading to the formation of these crystalline complexes, only few studies have provided insight into the mechanisms which initiate the formation of the metal carboxylate network (3)(4). For this purpose, in this work we focus on the changes that occur to the inorganic pigment particles and we specifically concentrate on the first phase of the pigment-binder reaction, monitoring the evolution of the leaching of metal ions in paints with a novel approach.

In detail, we use Electron Paramagnetic Resonance (EPR) spectroscopy coupled with Inductively Coupled Plasma-Optical Emission Spectrometry (ICP-OES), to investigate metal ion leaching and measure its temporal evolution in paint films. We have studied the first stage of the reaction between $\mathrm{ZnO}$ and linseed oil by using a paramagnetic probe diluted in $\mathrm{ZnO}$ crystallites. $\mathrm{Cu}^{11}\left(3 \mathrm{~d}^{9}\right.$ configuration) was chosen as a paramagnetic probe since its EPR spectrum strongly depends on its coordination and on the symmetry of its environment, hence providing information on changes of the $\mathrm{Cu}^{\prime \prime}$ environment.

$\mathrm{Cu}^{\prime \prime}$ has an imposed tetrahedral coordination in $\mathrm{ZnO}$, and the degenerate (or nearly degenerate) ${ }^{2} \mathrm{~T}_{2}$ ground state gives an EPR spectrum (with $g \|=0.74$ and $g \perp=1.53$ ) that is detectable only at low temperature (around $4 \mathrm{~K}$ ) 
(6). In contrast, if $\mathrm{Cu}^{\prime \prime}$ is free to achieve a six-fold or four-fold planar coordination, as would be the case for copper ions bound to carboxylates (in the following quoted as $\mathrm{Cu}^{\prime \prime}$-carboxylate complexes), the orbitally nondegenerate ground state would invariably give an EPR spectrum detectable at room temperature with typical $g$ factors equal to $\mathrm{g} \| \approx 2.25-2.40$ and $2.00<\mathrm{g} \perp<\mathrm{g} \|$ (see for example (7) (8)). For this reason, no Cu" EPR signal is expected at room temperature in $\mathrm{ZnO}$, whereas on the contrary, if mixing with binder induces a surface modification of $\mathrm{ZnO}$ and a metal ion extraction, the EPR spectrum of $\mathrm{Cu}^{\prime \prime}$-carboxylate complexes should appear.

In this work, we have monitored EPR over time in a mixture of $0.8 \%$ Cu-doped ZnO powder and linseed oil. The system mimics the progression of metal ion leaching in oil-based paint films and the EPR spectroscopy signal is used to monitor the evolution of metal ions. Finally, since EPR spectroscopy is silent with respect to the coordination of $\mathrm{Zn}$ ions (note that $\mathrm{Zn}$ " is diamagnetic), ICP-OES was used on the oil extracted from the $\mathrm{ZnO}: \mathrm{Cu}$ oil mixture, to confirm the migration of metal ions into the oil medium and to measure the concentrations of $\mathrm{Zn}$ and $\mathrm{Cu}$ ions.

\section{Materials and Methods}

\subsection{Materials}

The Cu-doped $\mathrm{ZnO}$ powders, labelled as $\mathrm{ZnO}: \mathrm{Cu}$, were synthesized by the co-precipitation method. The synthetic procedure is summarized as follows (9): $25 \mathrm{ml}$ solution (aq) of $1.49 \cdot 10^{-2} \mathrm{~mol}$ zinc acetate dihydrate (Sigma Aldrich, Milano, Italy) is mixed with $10 \mathrm{ml}$ water solution of $0.018 \cdot 10^{-2} \mathrm{~mol}$ copper acetate monohydrate ( $\geq 98 \%$ Sigma Aldrich, Milano, Italy). After the addition of $25 \mathrm{ml}$ of $\mathrm{KOH}\left(4.99 \cdot 10^{-2} \mathrm{~mol}\right)$ drop by drop under constant stirring, the mixture is heated at $80^{\circ} \mathrm{C}$ for 1 hour, until a precipitate is formed. The dispersion is washed and centrifuged (4000 rpm, $15 \mathrm{~min}$ ) twice with distilled water followed by ethanol to remove impurities. Finally, the $\mathrm{ZnO}: \mathrm{Cu}$ is calcined at $1000^{\circ} \mathrm{C}$ for $3 \mathrm{hrs}$ in a muffle furnace. In this way a $\mathrm{ZnO}: \mathrm{Cu}$ doped at $0.8 \%$ is obtained (as demonstrated by ICP-OES measurements). The same procedure was applied for the preparation of pure $\mathrm{ZnO}$, without the addition of the Cu-dopant. Historical recipes suggest heating (without boiling) of commercial cold-pressed linseed oil (supplied by Laverdure); in this work the linseed oil was heated for about 3 hours at 120 ${ }^{\circ} \mathrm{C}$ prior to mixing with pigment. It is noted that the starting linseed oil is pure and does not contain siccative compounds (such as litharge, $\mathrm{PbO}$ ) or other detectable metal ion impurities.

\subsection{Methods}

X-ray powder diffraction. The phase evolution of synthesized powders was monitored by X-ray diffractometry (XRD, (Panalytical Empyrean)) using a Bragg Brentano diffractometer and Cu-Ka1 radiation $(\lambda=0.154056 \mathrm{~nm})$. The $X$-ray diffraction patterns were collected at room temperature in the $10-70^{\circ} 2 \theta$ range with a scan step size of $0.02^{\circ}$.

Electron Paramagnetic Resonance Spectroscopy. For EPR spectroscopy measurements a sample containing $50 \mathrm{mg}$ of $\mathrm{ZnO}: \mathrm{Cu}$ powder mixed with $50 \mathrm{mg}$ of linseed oil was prepared. EPR spectra were recorded at room temperature with a Bruker Elexsys E500 spectrometer operating at X-band $(9.69 \mathrm{GHz})$ in a continuouswave mode and equipped with a Bruker 4122SHQE/0111 resonator. A magnetic field modulation at $100 \mathrm{kHz}$ was applied with an amplitude of $0.8 \mathrm{mT}$. The microwave power was $20 \mathrm{~mW}$. Simulations of EPR spectra were performed with the Easyspin software (10). The mass $m_{C u}$ of $\mathrm{Cu}^{\prime \prime}$ detected by EPR was estimated by comparing the intensities $I_{C u}$ of the $\mathrm{Cu}^{\text {Il }}$ spectra with the intensity $I_{\text {ref }}$ of a reference sample made of $40.0 \mathrm{mg}$ of $\mathrm{CaCuSi}_{4} \mathrm{O}_{10}$, corresponding to $6.4 \times 10^{19} \mathrm{Cu}^{\prime \prime}$ ions, mixed with $45 \mathrm{mg}$ of linseed oil, according to:

$$
m_{C u}=6.4 \times 10^{19} \times \frac{I_{C u}}{I_{\text {ref }}} \times \frac{M_{C u}}{N_{A}}
$$

with $M_{\mathrm{Cu}}=63.55 \mathrm{~g} \cdot \mathrm{mol}^{-1}$ the molar mass of $\mathrm{Cu}$ and $N_{\mathrm{A}}=6.02 \times 10^{23}$ the Avogadro number.

Inductively Coupled Plasma - Optical Emission Spectrometry (ICP-OES). The amount of Cu doping in the synthesized powder was evaluated by ICP-OES (Perkin Elmer Optima 3200) spectrometry as $0.8 \pm 0.1 \mathrm{w} \%$. ICP-OES measurements were further exploited to measure on leaching $(\mathrm{Zn}$ and $\mathrm{Cu}$ ) from the metal oxide pigment particles into the binding medium. The synthetized ZnO:Cu powder was mixed 1:1 (wt.) with linseed oil and kept in closed vessels in the dark. The obtained white paste was washed with diethyl ether and centrifuged 
at $4000 \mathrm{rpm}$ (Universal $32 \mathrm{R}$, Hettich Zentrifugen) to separate the organic components in supernatant from the ZnO powder. Linseed oil-ether solution was then analysed by ICP-OES after an acid water extraction (water/ $/ \mathrm{HO}_{3}$ ), multiple extractions were carried out to maximize the final recovery. The acidic conditions employed during extraction lead to the dissolution of any residual $\mathrm{ZnO}: \mathrm{Cu}$ particles. For this reason, $\mathrm{ZnO}: \mathrm{Cu}$ powder was analysed separately in the same conditions and used as a control to validate the efficiency of the centrifuging procedure in $\mathrm{ZnO}: \mathrm{Cu}$ particle removal, since the presence of residual $\mathrm{ZnO}: \mathrm{Cu}$ particles in the supernatant solution could invalidate the final $\mathrm{Zn}$ and $\mathrm{Cu}$ quantification. As a further control, the same measurement was carried out on pure linseed oil binder. A schematic representation of the entire procedure is summarized in Figure S1. The results obtained by different tests are consistent and the concentration of detected ions was above the detection limit of ICP-OES (approximately $0.05 \mu \mathrm{g} / \mathrm{l}$ ).

\section{Results}

\subsection{Monitoring reactions with a paramagnetic probe}

Figure $1(\mathrm{~A})$ shows the $\mathrm{X}$-band $(9.693 \mathrm{GHz})$ EPR spectra at room temperature recorded for the ZnO:Cu powder at different times after mixing it with linseed oil. The EPR spectrum of pure $\mathrm{ZnO}$ :Cu powder shows a very weak signal in the range 310-350 $\mathrm{mT}$. Its shape and position and the fact that it is detected at room temperature leads us to infer that the signal corresponds to $\mathrm{Cu}^{\prime \prime}$ in a distorted octahedral coordination (8), possibly in perturbed sites at the surface of the particles. In addition, signals from transition metal impurities present in the zinc acetate used during the synthesis of the powder can be observed: $\mathrm{Mn}^{\prime \prime}\left(3 \mathrm{~d}^{5}, \mathrm{~S}=5 / 2\right)$ is clearly recognizable thanks to the classical hyperfine pattern of the EPR transition between the $M_{S}=-1 / 2$ and $M_{S}=+1 / 2$ electron spin manifolds labelled by asterisks in Figure $1(\mathrm{~A})(11)$. In addition, a symmetrical line at $g=2.14$, labelled by a cross, is typical of $\mathrm{Ni}^{\prime \prime}$ in octahedral symmetry $\left(3 \mathrm{~d}^{8},{ }^{3} \mathrm{~A}_{2}\right.$ ground state) (8), which could correspond to the cation interstitial site in the $\mathrm{ZnO}$ structure. Our interpretation of a six-fold coordinated $\mathrm{Nil}$ for the line at $g=2.14$ is supported by a recent study of Ni-doped $\mathrm{ZnO}$ nanopowders reporting a signal at $g=2.19$ attributed to $\mathrm{Ni}^{\text {il }}$ in octahedral coordination (12). Indeed, $\mathrm{Ni}^{11}$ with $3 \mathrm{~d}^{8}$ configuration has an integer spin $\mathrm{S}=1$. Generally, ions with integer non-zero spin are not detectable in EPR at X-band unless the Zero-Field Splitting (ZFS) interaction is very small (8). This can however be the case in a high symmetry environment such as the interstitial octahedral site of ZnO. A possible $\mathrm{Ni}^{\mathrm{il}}$ substituting in the tetrahedral cation site of $\mathrm{ZnO}$ with a then a degenerate orbital state $\left({ }^{3} \mathrm{~T}_{1}\right)$ should not be detectable at room temperature, owing to spin-orbit coupling, which would induce a strong relaxation broadening of its EPR signal. An undetermined signal at $g=2.55$ labelled by a square in Figure $1(A)$ is also observed.

Figure 1 - (A) Room temperature X-band (9.693 GHz) EPR spectra of the ZnO:Cu powder sample (black line) compared with the spectra of the same powder mixed with linseed oil, at different time-points. (B) Experimental and simulated EPR spectra of ZnO:Cu mixed with linseed oil after ten days and simulated contributions of $\mathrm{Cu}^{\prime \prime}$, $\mathrm{Mn}^{\prime \prime}\left({ }^{*}\right), \mathrm{Ni}^{\prime \prime}(\dagger)$ and undetermined species $(g=2.55)$.

Linseed oil does not present any paramagnetic signal at room temperature. The EPR spectrum of $\mathrm{ZnO}: \mathrm{Cu}$ is not modified immediately after mixing with linseed oil, as only $\mathrm{Mn}^{\prime \prime}, \mathrm{Ni}^{\mathrm{ll}}$ and the $g=2.55$ signals are detected and the weak $\mathrm{Cu}^{\prime \prime}$ signal is unchanged. Over time the $\mathrm{Cu}^{\prime \prime}$ signal grows progressively in the 335-345 mT region, achieving significant intensity values after a few hours (Figure 1(A)). As this signal increases at different time delays after mixing with oil, we conclude that it is due to the conversion of EPR silent $\mathrm{Cu}^{\prime \prime}$ in four-fold tetrahedral environment into EPR active $\mathrm{Cu}^{\prime \prime}$ in six-fold or square-planar environment. This EPR signal is simulated with spin Hamiltonian parameters $\left(g_{z}=2.3, g_{x}=2.045, g_{y}=2.1, A_{z}=483 \mathrm{MHz}, A_{x}=27 \mathrm{MHz}, A_{y}=88 \mathrm{MHz}\right.$ for $\left.{ }^{63} \mathrm{Cu}\right)$, as shown in Figure 1(B). Values of the spin-Hamiltonian and, more specifically, of the hyperfine parameters $A_{x}, A_{y}$ and $A_{z}$ are fairly inaccurate due to the lack of spectral resolution but are in the range of values for $\mathrm{Cu}^{\prime \prime}$ coordinated to oxygen ions (7). All simulation parameters, including $g$ - and A-strain (effect of ligand field disorder), are given in Table S1.

The variation with time of the amount of $\mathrm{Cu}^{\prime \prime}$ detected by EPR is depicted in Figure 2 (filled red circles). The increase in EPR active $\mathrm{Cu}^{\prime \prime}$ is very steep in the first hours after mixing, but after a few days the growth rate significantly decreases, and the intensity reaches saturation level (note that the binding oil is still liquid at this time). Small variations in the $\mathrm{Ni}^{\prime \prime}$ and $\mathrm{Mn}{ }^{\prime \prime}$ EPR intensities are within experimental errors.

Figure 2 - Ratio of the mass of released cation to the initial mass of $\mathrm{ZnO}: \mathrm{Cu}$ in linseed oil, at different time delays after mixing and detected either by EPR or by ICP-OES. Red line: simulation with equation (2). 


\subsection{Concentration of metal ions extracted in oil}

In order to demonstrate that $\mathrm{Zn}^{\|}$ions are also released in the investigated system, together with the leaching of $\mathrm{Cu}^{\text {Il }}$ ions, we have tested the presence of both metal ions in the oil extracted from ZnO:Cu-linseed oil with ICPOES. In order to employ ICP analyses on isolated linseed oil, the $\mathrm{ZnO}: \mathrm{Cu}$ is dispersed in linseed oil forming a white paste, which is then washed with diethyl ether and centrifuged (Figure S1). This procedure allows the separation of the organic components in supernatant from the $\mathrm{ZnO}: \mathrm{Cu}$ powder, allowing us to measure the metal content released in the extracted linseed oil. For pure linseed oil, a residual $\mathrm{Zn}$ content of $0.18 \mathrm{mg} . \mathrm{L}^{-1}$ is detected. In the case of $\mathrm{ZnO}: \mathrm{Cu}$ powder alone (not mixed with oil) we have detected residual amounts of 62 mass ppm and 5 mass ppm for $\mathrm{Zn}$ and $\mathrm{Cu}$, respectively. $\mathrm{Zn}$ and $\mathrm{Cu}$ ions in the $\mathrm{ZnO}: \mathrm{Cu}$ powder mixed with linseed oil have been measured up to 7 days after mixing and results are shown in Figure 2. ICP-OES analysis reveals a significant increase in the concentration of both $\mathrm{Cu}$ and $\mathrm{Zn}$ ions after only few minutes of mixing $\mathrm{ZnO}: \mathrm{Cu}$ with linseed oil, followed by saturation. $\mathrm{Zn}$ or $\mathrm{Cu}$ released in oil after 3 days correspond to about $0.1 \%$ and $0.06 \%$, respectively, of $\mathrm{Zn}$ and $\mathrm{Cu}$ in the $\mathrm{ZnO}: \mathrm{Cu}$ powder.

\section{Discussion}

EPR analyses show that the environment of $\mathrm{Cu}^{\prime \prime}$ changes due to the reaction of ZnO:Cu with linseed oil. The $\mathrm{Cu}^{\prime \prime}$ metal ions switch from tetrahedral $\mathrm{CuO}_{4}$ coordination to octahedral $\mathrm{CuO}_{6}$ or $\mathrm{CuO}_{4}$ planar coordination. It is worth stressing that the detected EPR spectrum of $\mathrm{Cu}^{\text {II }}$ does not show the usual four-line hyperfine pattern of the parallel component of the spectrum. The "smoothing" is due to a strong broadening of the hyperfine lines, typically produced by ligand field strain and disorder that in turn induces a distribution of $g$ and $A$ parameter values, as reported also in (13). Although the measured $g$ and $A$ parameter values are typical of $\mathrm{Cu}^{\text {" }}$ with oxygen ligands (7), evidence of strong disorder suggests that the EPR spectrum is most probably the sum of several types of $\mathrm{Cu}^{\prime \prime}$ environments, which differ in their symmetry and/or in the nature of the coordinating ligands. We propose at least two different situations to account for this observation. The first one is the coordination of $\mathrm{Cu}^{\prime \prime}$ at the perturbed $\mathrm{ZnO}$ surface. In this case, the oxygen ligands of the $\mathrm{Cu}^{\prime \prime}$ complex can belong to both $\mathrm{ZnO}$ and oil carboxylate groups with different configurations. For example, $\mathrm{Cu}$ " can be bound with four O ligands from $\mathrm{ZnO}$ and two additional ligands from carboxylates, or with two ligands from $\mathrm{ZnO}$ and four from carboxylates. In the second situation, $\mathrm{Cu}^{\prime \prime}$ ions are extracted from $\mathrm{ZnO}$ and bound only to carboxylate groups. Hence, the superposition of these different spectra with different $g_{z}$ and $A_{z}$ parameters considerably distorts the low field part of the spectrum (14).

The evolution of the amount of $\mathrm{Cu}^{\prime \prime}$ detected by EPR and illustrated in Figure 2, can be empirically interpolated with a power law

$$
M(t) \cong M_{0}+A t^{n}
$$

with $M_{0} \cong 3.9 \mathrm{ppm}$ (the initial $\mathrm{Cu}^{\prime \prime}$ amount corresponding to $\mathrm{Cu}^{\prime \prime}$ at the surface of the raw particles), $A \cong 6 \mathrm{ppm}$. $\mathrm{h}$ ${ }^{n}$ and $n \cong 0.30$. It follows that the kinetics of the released $\mathrm{Cu}^{\prime \prime}$ ions deviates form a simple first order kinetics, which would give an exponential rise. In metal oxide dissolution, such a power law can be affected by several factors such as particle shape (15), heterogeneous diffusion (16) and chemical processes at the solid/liquid interface (17). An important result of our measurement is that the complete dissolution of $\mathrm{Cu}^{\text {"l }}$ ions is not reached even after several days in oil. One possible explanation could be related to a combination of $\mathrm{pH}$ effect and passivation phenomenon. Acidity is known to be a major parameter controlling dissolution (17). In an acidic medium, as is the case of linseed oil, $\mathrm{Zn}-\mathrm{O}$ bonds are weakened by adsorption of carboxylic $-\mathrm{COOH}$ groups. Acid groups bond to $\mathrm{Zn}-\mathrm{O}$ via dissociative adsorption (bidentate mode) and hydrogen ions migrate from $-\mathrm{COOH}$ to oxygen sites. Therefore, the dissolution occurs rapididly directly after mixing ZnO particles with oil, i.e. when $\mathrm{pH}$ is low. As dissolution proceeds, protons are consumed, and $\mathrm{pH}$ increases. In this process, the dissolution slows because the $\mathrm{ZnO}$ surface approaches its zero-charge point (neutral surface condition) and becomes less reactive (17). In addition, COO- groups covalently bind to the ZnO surface and lead to passivation (18) (19), halting ion leaching. 
It can be noticed that the rate of copper release detected by ICP-OES is much faster than that detected by EPR (Fig.2). A possible explanation of this effect is to that $\mathrm{Cu}^{\prime \prime}$ are first liberated in the form of EPR silent tetrahedral complexes, which then slowly convert to square planar or (and) six-fold coordinated EPR active complexes. For example, this may happen if the reaction with binding oil proceeds first by extracting very small (nanometer size) $\mathrm{ZnO}$ aggregates, containing $\mathrm{Cu}^{\prime \prime}$ in tetrahedral coordination, which are then completely dissolved in the binding oil, as depicted in Figure 3. If these particles are small enough to remain in the liquid phase after centrifugation (Fig. 3), ICP-OES detects all $\mathrm{Cu}^{\text {II }}$ produced during the two steps, i.e. both EPR silent $\mathrm{Cu}^{\prime \prime}$ still bound to small $\mathrm{ZnO}$ particles and the $\mathrm{Cu}^{\prime \prime}$ ions dissolved in oil. Instead, EPR can detect only $\mathrm{Cu}^{\prime \prime}$ produced during the second step. The dissolution of $\mathrm{ZnO}$ in oil thus shares similarities with the dissolution of oxide particles in aqueous solutions that lead to a release of metal ions in the liquid phase (20) (21) (22) (23).

Figure 3 - (a) Pristine ZnO particle containing EPR silent Cull (black dots). (b) Partial dissolution of smaller ZnO particles and releasing EPR active $\mathrm{Cu}^{\prime \prime}$ in oil (red dots). (c) Full dissolution of the smaller particles and formation of outer passivation layer (orange) which hinders the dissolution phenomenon.

Because of the partial dissolution process, the $\mathrm{ZnO}$ surface structure is strongly modified after the dispersion in oil medium. This should in turn change the photoluminescence properties of $\mathrm{ZnO}$ grains. Indeed, in other research (24) (25) we have clearly detected an enhancement of the photoluminescence intensity from trap state levels of zinc oxide mixed with oil with respect to unmixed zinc oxide. This change can be attributed to the adsorption of carboxylate groups to the $\mathrm{ZnO}$ surface, which affects the electronic structure at $\mathrm{ZnO}$ surface and its luminescence properties (26). The changes in luminescence have been shown to be significant in the first days after mixing of pigment powders and oils, but stable in time once the paint film become dried (24) (25).Results of EPR analysis allow us to better explain this evolution in time of the photoluminescence signal as a consequence of the passivation of $\mathrm{ZnO}$ which reaches a steady-state after a few days after mixing.

\section{Conclusion}

In this work, we applied for the first time EPR spectroscopy combined with ICP-OES to probe the release of metal ions in paint films, and in particular we considered the case of ZnO particles dispersed in oil medium. The most remarkable results are related to the determination of the kinetics of the metal ion leaching. Firstly, the results show that the pigment particles dissolve in drying oil following a trend comparable to dissolution of metal oxides in aqueous solutions, which is steep in the first stage and then slows because the passivation of the $\mathrm{ZnO}$ surface by carboxylate groups. A steady-state condition is reached in ten days, significantly preceding the complete hardening of the paint film.

The second important result regards the strong tendency of metal-based pigments to react with acid groups of drying oils. The latter are governed by the surface pigment reactivity towards the adsorption of acids and by the tendency of the pigment particles to dissolve in the oil medium. The reactivity of metal-based pigments can be controlled by adjusting their surface characteristics, by applying a surface coating, or by controlling the $\mathrm{pH}$ of the mixture in order to limit the metal ion leaching.

The method applied in this study for monitoring the metal ion leaching in paint mixing could be employed to study the effect of particle size on reactivity, and to investigate other oil-based paints, in which metal ion leaching phenomena and migration of metal complexes are implicated in paint degradation, as in the case of lead white oil paint.

\section{Acknowledgment}

Research was partially funded by the Erasmus+ Traineeship program.

\section{Conflicts of interest}

There are no conflicts to declare.

\section{References}


1. Osmond G, Boon JJ, Puskar L, Drennan J. Metal stearate distributions in modern artists' oil paints: Surface and cross-sectional investigation of reference paint films using conventional and synchrotron infrared microspectroscopy. Appl Spectrosc. 2012;66(10):1136-44.

2. Gabrieli F, Rosi F, Vichi A, Cartechini L, Buemi LP, Kazarian SG, et al. Revealing the nature and distribution of metal carboxylates in Jackson Pollock's alchemy (1947) by micro-attenuated total reflection FT-IR spectroscopic imaging. Anal Chem. 2017;

3. Hermans JJ, Keune K, Van Loon A, ledema PD. The crystallization of metal soaps and fatty acids in oil paint model systems. Phys Chem Chem Phys. 2016;18(16):10896-905.

4. Baij L, Hermans JJ, Keune K, ledema P. Time-Dependent ATR-FTIR Spectroscopic Studies on Fatty Acid Diffusion and the Formation of Metal Soaps in Oil Paint Model Systems. Angew Chemie - Int Ed. 2018;57(25):7351-4.

5. Mecklenburg MF, Tumosa CS, Vicenzi EP. The Influence of Pigments and lon Migration on the Durability of Drying Oil and Alkyd Paints. New Insights into Clean Paint Proc from Clean 2010 Int Conf Univ Politec Val Museum Conserv Inst. 2013;

6. Dietz RE, Kamimura H, Sturge MD, Yariv A. Electronic structure of copper impurities in ZnO. Phys Rev. 1963;132(4):1559-69.

7. Peisach J, Blumberg WE. Structural implications derived from the analysis of electron paramagnetic resonance spectra of natural and artificial copper proteins. Arch Biochem Biophys. 1974;165(2):691-708.

8. de Boer E. Electron Paramagnetic Resonance. Elementary Theory and Practical Applications, J.A. Weil, J.R. Bolton and J.E. Wertz, John Wiley, U.S.A., 1994 xxi + 568 pp., £66.00, ISBN 0-471-57234-9. Recl des Trav Chim des Pays-Bas. 2010;

9. Baskar G, Gurugulladevi A, Nishanthini T, Aiswarya R, Tamilarasan K. Optimization and kinetics of biodiesel production from Mahua oil using manganese doped zinc oxide nanocatalyst. Renew Energy. 2017;103:641-6.

10. Stoll S, Schweiger A. EasySpin, a comprehensive software package for spectral simulation and analysis in EPR. J Magn Reson. 2006;

11. Li GL, Wu SY, Hu XF, Kuang MQ. Theoretical studies on the spin Hamiltonian parameters and local environment of Mn2+ in ZnO nanocrystal and bulk. Phys B Condens Matter [Internet]. 2015 Jun 1 [cited 2019 Feb 3];466-467:64-7. Available from:

https://www.sciencedirect.com/science/article/pii/S0921452615300016

12. Babu B, Sundari GR, Ravindranadh K, Yadav MR, Ravikumar RVSSN. Structural, spectroscopic and magnetic characterization of undoped, Ni 2+ doped ZnO nanopowders. J Magn Magn Mater [Internet]. 2014 Dec 1 [cited 2019 Feb 3];372:79-85. Available from: https://www.sciencedirect.com/science/article/pii/S0304885314006775

13. Gourier D, Vivien D, Thery J, Livage J, Collongues R. ESR of copper-doped $\beta$-alumina localization of Cu2+ and linewidth effect. Phys Status Solidi. 1978;45(2):599-606.

14. Marquardt CL, Giuliani JF, Gliemeroth G. A study of copper ions in silver-halide photochromic glasses. J Appl Phys. 1977;48(9):3669-79.

15. Kline WE, Fogler HS. Dissolution kinetics: The nature of the particle attack of layered silicates in HF. Chem Eng Sci. 1981;

16. Macheras $\mathrm{P}$, Dokoumetzidis A. On the heterogeneity of drug dissolution and release. Pharmaceutical Research. 2000.

17. Stumm W. Reactivity at the mineral-water interface: Dissolution and inhibition. Colloids Surfaces A Physicochem Eng Asp. 1997;

18. Taratula O, Galoppini E, Wang D, Chu D, Zhang Z, Chen H, et al. Binding studies of molecular linkers to 
ZnO and MgZnO nanotip films. J Phys Chem B. 2006;

19. Moreira NH, Da Rosa AL, Frauenheim T. Covalent functionalization of ZnO surfaces: A density functional tight binding study. Appl Phys Lett. 2009;

20. Yoon TH, Johnson SB, Brown GE. Adsorption of Organic Matter at Mineral/Water Interfaces. IV. Adsorption of Humic Substances at Boehmite/Water Interfaces and Impact on Boehmite Dissolution. Langmuir. 2005;

21. Sakallioglu T, Bakirdoven M, Temizel I, Demirel B, Copty NK, Onay TT, et al. Leaching of nano-ZnO in municipal solid waste. J Hazard Mater. 2016;

22. Petrochenko PE, Zhang Q, Bayati R, Skoog SA, Phillips KS, Kumar G, et al. Cytotoxic evaluation of nanostructured zinc oxide (ZnO) thin films and leachates. Toxicol Vitr. 2014;28(6):1144-52.

23. Bian SW, Mudunkotuwa IA, Rupasinghe T, Grassian VH. Aggregation and dissolution of $4 \mathrm{~nm} Z \mathrm{nO}$ nanoparticles in aqueous environments: Influence of $\mathrm{pH}$, ionic strength, size, and adsorption of humic acid. Langmuir. 2011;27(10):6059-68.

24. Artesani A, Gherardi F, Nevin A, Valentini G, Comelli D. A photoluminescence study of the changes induced in the zinc white pigment by formation of zinc complexes. Materials (Basel). 2017;10(4).

25. Artesani A, Gherardi F, Mosca S, Alberti R, Nevin A, Toniolo L, et al. On the photoluminescence changes induced by ageing processes on zinc white paints. Microchem J. 2018;

26. Zhang Z, Yates JT. Band Bending in Semiconductors: Chemical and Physical Consequences at Surfaces and Interfaces. Vol. 112, Chemical Reviews. 2012. p. 5520-51. 